\title{
Influence of Treatment Intensification on A1c in Patients with Suboptimally Controlled Type 2 Diabetes After 2 Oral Antidiabetic Agents
}

\author{
Kibum Kim, BPharm, MSc, PhD; Sudhir Unni, PhD, MBA; Carrie McAdam-Marx, RPh, MS, PhD; \\ Sheila M. Thomas, PharmD; Kimberly L. Sterling, PharmD, MS; Cody J. Olsen, PharmD, BCPS; \\ Bryan Johnstone, PhD; Matt Mitchell, PharmD, MBA, FAMCP; and Diana Brixner, RPh, PhD, FAMCP
}

\begin{abstract}
BACKGROUND: In the United States, more than $50 \%$ of patients with type 2 diabetes mellitus (T2DM) have hemoglobin A1c (A1c) levels that fail to achieve the recommended target of $<7.0 \%$. Of these, $30 \%-45 \%$ have an A1C $>9.0 \%$, the threshold for poorly controlled T2DM per National Committee for Quality Assurance (NCQA) measures. Treatment inertia is a known challenge. However, recent treatment intensification patterns and outcomes after treatment fails 2 classes of oral antidiabetic agents (OADs) are not well understood.
\end{abstract}

OBJECTIVE: To characterize treatment intensification patterns and glycemic control outcomes in patients with $A 1 C \geq 7.0 \%$ on 2 OADs.

METHODS: A retrospective cohort study was conducted in patients with T2DM from a regional health plan claims dataset augmented with A1C results between January 1, 2010, and March 31, 2017. Patients were identified with an $A 1 c \geq 7.0 \%$ (baseline), while on $20 A D s$, and whose treatment was intensified with basal/biphasic insulin (insulin), glucagon-like peptide-1 receptor antagonist (GLP-1RA), or a third OAD within 365 days after the baseline $A 1 \mathrm{c} \geq 7.0 \%$. Patients had at least $1 \mathrm{~A} 1 \mathrm{c}$ value $60-365$ days (follow-up period) after treatment intensification. The proportion of patients with an $\mathrm{A} 1 \mathrm{c}<\mathbf{2 . 0 \%}$ and $<9.0 \%$ at follow-up were identified by therapeutic intensification strategy. Odds ratios for achieving A1c $<7.0 \%$ and $<9.0 \%$ were calculated.

RESULTS: 1,226 patients were included in the analysis, and $33.5 \%$ of the patients had a baseline $A 1 \mathrm{C} \geq 9.0 \% .24 \%$ of patients received insulin; $16 \%$ received GLP-1RA; and $60 \%$ received a third OAD for the treatment intensification. Overall, $26.0 \%$ achieved A1c $<7.0 \%$ and $76.1 \%$ of patients achieved $<9.0 \%$, with a median follow-up of 119 days. The proportion of patients intensified with insulin who had an $A 1 c \geq 9.0 \%$ at follow-up was $34.6 \%$ versus $53.2 \%$ at baseline $(P<0.01)$. The corresponding percentages for those intensified with a GLP-1RA and OAD were $21.6 \%$ versus $27.1 \%$ $(P=0.24)$ and $20.1 \%$ versus $27.3 \%(P<0.01)$. After controlling for baseline characteristics, the odds ratio $(95 \% \mathrm{Cl})$ of achieving $\mathrm{A} 1 \mathrm{C}<7.0 \%$ and $<9.0 \%$ was 2.05 (1.45-2.90) for GLP-1RA and $0.92(0.61-1.40)$ for OAD. The association between goal attainment and GLP-1RA versus OAD intensification was influenced by the time to the A1c follow-up and baseline A1c.

CONCLUSIONS: Treatment intensification was associated with improved glycemic control in patients after therapy failed 2 OADs. Patients with higher A1c at baseline were likely to initiate insulin, which was associated with a greater drop in A1c. GLP-1RA was associated with a higher likelihood of achieving NCQA-suggested glycemic control compared with a third OAD. However, the association varied by the follow-up period. These findings are important to health plans seeking to improve patient outcomes as reflected in high performance on NCQA diabetes quality measures by promoting effective and timely treatment intensification.

J Manag Care Spec Pharm. 2019;25(3):314-22

Copyright $\odot 2019$, Academy of Managed Care Pharmacy. All rights reserved.

\section{What is already known about this subject}

Switching to or addition of an antidiabetic agent in a third class leads to better outcomes across varying levels of hemoglobin Alc (Alc) goals in patients who already failed to achieve glycemic control on metformin alone or in combination with an oral antidiabetic agent (OAD)

According to the National Committee for Quality Assurance (NCQA) Healthcare Effectiveness Data and Information Set (HEDIS) measures, the proportion of adult patients meeting Alc goals of $<7.0 \%$ to $<9.0 \%$ indicates the quality of comprehensive diabetes care.

\section{What this study adds}

This study identified the pattern of treatment intensification in patients whose Alc was insufficiently controlled on OADs in 2 classes: Patients continued to OAD in a third class more often than insulin or glucagon-like peptide-1 receptor antagonist (GLP-1RA)

The proportion of patients meeting Alc goals of $<7.0 \%$ to $<9.0 \%$ increased after the third treatment intensification, which was consistently observed across the 3 intensification groups: insulin, GLP-1RA, and third OAD.

The odds of achieving Alc $<7.0 \%$, after adjusting for baseline Alc and other potential confounders, were significantly higher in the GLP-1RA group relative to the third OAD group

$\mathrm{T}$ ype 2 diabetes mellitus (T2DM) is a chronic condition that requires long-term management and self-care. In 2012, the medical cost of treating a patient with diabetes averaged $\$ 13,700$ annually, which was 2.3 times the expenditure for patients without diabetes. ${ }^{1}$ Given that the prevalence of diabetes in the United States has reached 9.4\%, with more than $90 \%$ of these patients diagnosed with T2DM, the economic burden of diabetes in 2012 was $\$ 245$ billion including medical costs and lost productivity. ${ }^{2}$ Inadequate glycemic control contributes to a substantial increase in the risk of vascular complications and potentially leads to blindness or premature death. ${ }^{3-5}$ Consequently, T2DM patients who have insufficiently 
controlled hyperglycemia are expected to use more medical resources. ${ }^{6}$

The National Committee for Quality Assurance (NCQA) has compiled a comprehensive diabetes care quality measurement set using Healthcare Effectiveness Data and Information Set (HEDIS) measures and has promoted high-quality care for patients through the Diabetes Recognition Program. ${ }^{7}$ Current comprehensive diabetes care measures assess 3 levels of hemoglobin Alc (Alc) as indicators of population-level diabetes control and diabetes quality of care. The measures include the proportion of adult patients who are candidates for aggressive control as Alc $<7.0 \%$, proportion with $\mathrm{Alc}<8.0 \%$ as a level appropriate for most patients to be below, and proportion with Alc $>9.0 \%$ as poorly controlled glycemia. ${ }^{7}$

The NCQA diabetes measures have been used to evaluate the performance of clinical practice and patient education in multiple studies. ${ }^{8-10}$ Patients with $\mathrm{Alc}<7.0 \%$ were expected to live with a manageable risk of complications. ${ }^{11}$ However, it is estimated that around 50\% of patients with diabetes in the United States have Alc levels that failed to achieve sustained glycemic control of $<7.0 \%$, and about $15 \%$ of patients had Alc $\geq 9.0 \%$, an indicator of poorly controlled hyperglycemia. ${ }^{12}$

Existing studies have demonstrated that shorter clinical inertia in patients whose Alc is not sufficiently controlled is potentially associated with a lower follow-up Alc value. In patients with newly diagnosed T2DM whose treatment failed metformin (MET) monotherapy, time to Alc goal attainment was shorter among patients who received early treatment intensification regardless of the Alc goal. ${ }^{13} \mathrm{Fu}$ et al. $(2016,2017)$ also analyzed change in Alc associated with treatment intensification among T2DM patients with Alc $>8.0 \%$ and demonstrated a positive association between treatment intensification and Alc reduction. ${ }^{14,15}$ Practice guidelines recommend treatment intensification, including switching to or addition of an antidiabetic agent in patients with insufficient glycemic control, based on the patient's cardiovascular risk and the drug's ability to lower Alc. ${ }^{16,17}$

Previous studies included patients on MET monotherapy or MET combined with another oral antidiabetic agent (OAD). Therefore, the identified pattern of treatment intensification and the outcomes have limited generalizability to patients receiving other OAD combinations. ${ }^{13-15}$ Levin et al. (2014) described treatment patterns in patients whose glycemia was insufficiently controlled on 2 OADs. ${ }^{18}$ However, the Levin study included treatment intensification between January 2000 and March 2009; therefore, recent patterns of antidiabetic agent use, specifically for glucagon-like peptide-1 receptor antagonist (GLP-1RA) after failure on 2 OADs, have not been studied. ${ }^{18}$
Given the limitations of previous studies, there is a need for a description of recent trends in treatment intensification after treatment fails 2 OADs. Further, assessment of the NCQA-HEDIS criteria to determine the Alc outcome for an observational study will facilitate better interpretation of quality measures in clinical practice, particularly within a managed care setting. The objectives of this study were to (a) characterize treatment intensification patterns and time to treatment intensification in patients with T2DM whose diabetes was insufficiently controlled per Alc $>7.0 \%$ on 2 OADs; (b) assess diabetes control associated with treatment intensification per NCQA-HEDIS measures; and (c) compare glycemic control between 3 treatment intensification strategies-adding basal/ biphasic insulin (insulin), GLP-1RA, or a third OAD.

\section{Methods}

\section{Study Design and Analytic Cohort}

This was a retrospective observational study using deidentified medical and pharmacy claims and Alc test results from SelectHealth, a regional managed care organization, between January 1, 2010, and March 31, 2017. SelectHealth operates in the Intermountain Region of the United States and collects medical and pharmacy claims for approximately 800,000 patients. The enrollees are predominantly from commercial lines but include some Medicaid and Medicare Advantage beneficiaries. Therefore, the population of this study is younger than a typical T2DM population. SelectHealth also captures Alc values for patients with T2DM, promoted by a diabetes incentive program for providers. This research was deemed exempt from the University of Utah's institutional review board (IRB) review (January 27, 2017) and was approved by the Intermountain Healthcare IRB expedited review (May 25, 2017).

The analytic cohort consisted of patients who had a record of $\mathrm{Alc} \geq 7.0 \%$ (i.e., baseline $\mathrm{Alc}$ ) after receiving prescriptions for 2 different $\mathrm{OAD}$ classes, including fixed-dose combinations. The baseline period was up to 365 days before the baseline Alc date, during which demographic information and clinical characteristics were captured. Charlson Comorbidity Index (CCI) and Diabetes Complication Severity Index (DCSI) scores were calculated using a published algorithm. ${ }^{19,20}$ To confirm insufficient response to therapy, the second OAD agent was required to have been dispensed at least 60 days before the baseline Alc date. Included patients had a diagnosis of T2DM (International Classification of Diseases, Ninth/Tenth Revision, Clinical Modification codes 250.x0 or 250.x2; E11x) on at least 2 different days during the 365-day baseline period; those with a diagnosis for type 1 diabetes or gestational diabetes were excluded. Also excluded were patients who received insulin or other injectable antidiabetic agents, including GLP-1RA or pramlintide, during the baseline period. Patients who filled pramlintide, an injectable amylin analogue, on the date of treatment intensification were also excluded. 


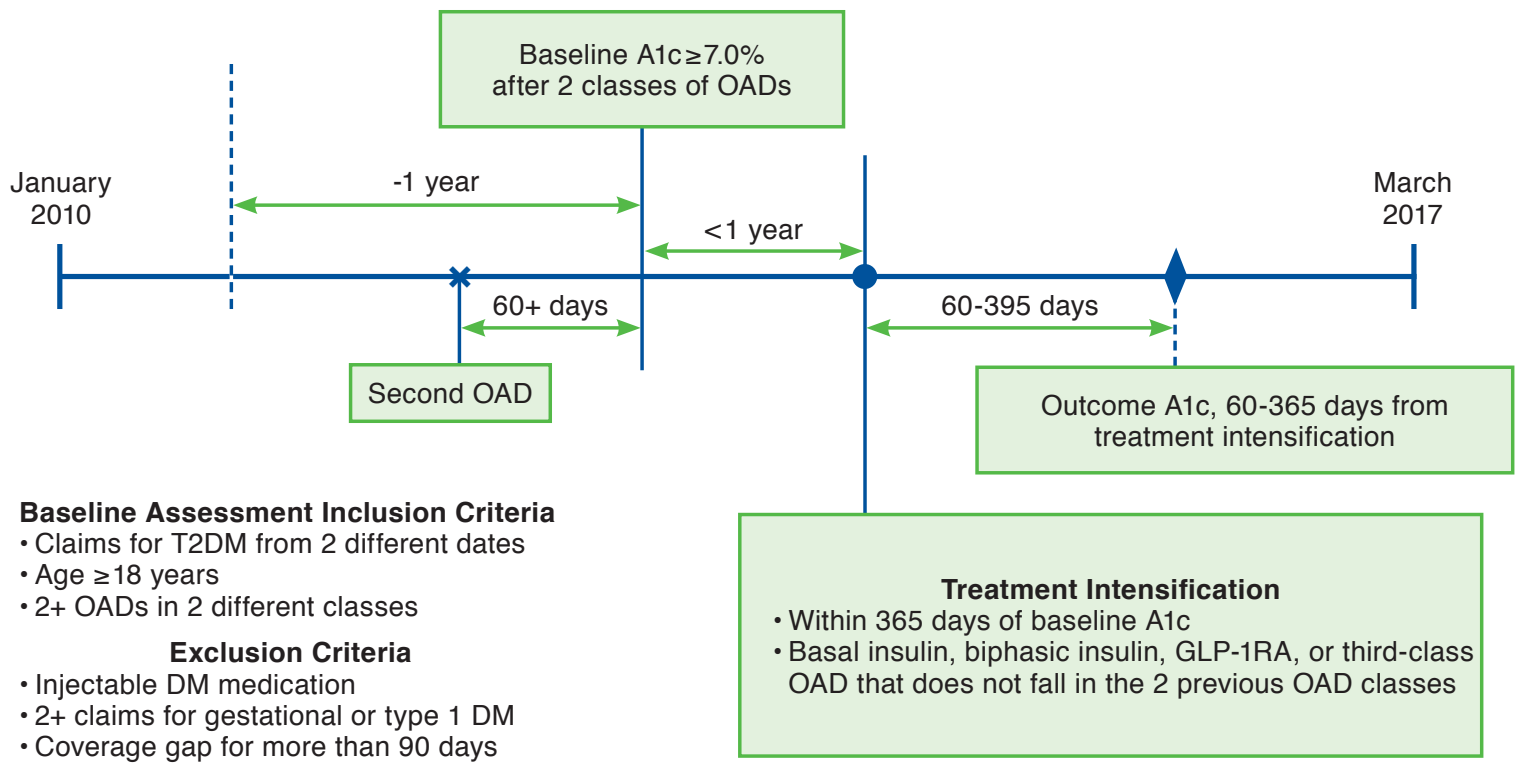

Alc=hemoglobin A1c; DM = diabetes mellitus; GLP-1RA=glucagon-like peptide-1 receptor antagonist; $O A D=$ oral antidiabetic agent; $T 2=$ type 2.

Pramlintide is specifically indicated for T2DM without optimal glycemic control despite an insulin therapy and therefore would not qualify as a treatment intensification option in our analysis. Included patients were required to have continuous enrollment, defined as constant coverage or a gap of no more than 90 days over the baseline period (Figure 1). The influence of patients having a coverage gap of $30+$ or $60+$ days was assessed as a sensitivity analysis. However, these shorter, allowed gaps had minimal effect on cohort size ( $\sim 3 \%$ difference; see Appendix A, available in online article).

\section{Treatment Intensification and Outcomes}

Treatment intensification was defined as a prescription for the first antidiabetic agent that differed from the 2 previous OAD classes and was classified by the type of antidiabetic agentinsulin, GLP-1RA, or a third OAD including thiazolidinedione (TZD), sulfonylurea (SU), alpha-glucosidase inhibitor, dipeptidyl peptidase 4 inhibitor (DPP-4), or sodium-glucose cotransporter inhibitor (SGLT2). Patients had to have a treatment intensification on or within 365 days from the baseline Alc date. Time (in days) from baseline Alc date to the treatment intensification date was also captured.

The primary outcome for the treatment intensification was glycemic control based on the first Alc measure and was captured between 60 and 365 days after treatment intensification (Figure 1). Glycemic control was defined as Alc $<7.0 \%$ and $<9.0 \%$, the lowest and highest Alc criteria from the
NCQA-HEDIS performance measures for diabetes care. The change in Alc from baseline to follow-up period was evaluated as a secondary outcome.

\section{Statistical Analysis}

All baseline and outcomes variables were descriptively analyzed by treatment intensification group. Continuous variables including age, morbidity index scores, Alc measures, number of days to treatment intensification, and the number of days to follow-up Alc were summarized using mean and standard deviation. Frequencies and percentages were reported for categorical variables that included gender, age over 65 years, geographic location (by state), type of insurance, baseline Alc $>9.0 \%$, and grouped comorbidity index scores.

Statistical significance was tested for insulin versus noninsulin, GLP-1RA versus third OAD intensification, and across the 3 groups using either Student's t-test or analysis of variance (ANOVA) for continuous variables and a chi-square test for categorical variables. When the expected number of patients in a cell of a 2-by-2 table was less than 5, the Fisher's exact test was used for the categorical variable.

The odds of achieving Alc $<7.0 \%$ and $<9.0 \%$ with GLP1RA intensification compared with intensification with a third OAD was assessed using a logistic regression model. Iterations of the model included baseline Alc $<9.0 \%$ versus $\geq 9.0 \%$ as a covariate, as well as with the cohort stratified by Alc $<9.0 \%$ versus $\geq 9.0 \%$, in order to account for the influence of baseline 
Influence of Treatment Intensification on A1c in Patients with

Suboptimally Controlled Type 2 Diabetes After 2 Oral Antidiabetic Agents

TABLE 1 Baseline Characteristics by the Type of Treatment Intensification

\begin{tabular}{|c|c|c|c|c|c|c|}
\hline & \multirow{2}{*}{$\begin{array}{c}\text { Insulina }^{\mathrm{a}} \\
\mathrm{n}=295\end{array}$} & \multirow{2}{*}{$\begin{array}{c}\text { GLP-1RA }^{\mathrm{b}} \\
\mathrm{n}=199\end{array}$} & \multirow{2}{*}{$\begin{array}{l}\mathrm{OAD}^{\mathrm{c}} \\
\mathbf{n}=732\end{array}$} & \multicolumn{3}{|c|}{$P$ Value } \\
\hline & & & & 3 Arms & Insulin vs. Other & GLP-IRA vs. OAD \\
\hline Age, mean (SD) & $53.93(11.22)$ & $52.24(10.34)$ & $55.47(9.98)$ & 0.22 & 0.25 & $<0.01$ \\
\hline$<65(\%)$ & 86.8 & 92.0 & 85.7 & 0.07 & 0.98 & 0.03 \\
\hline$\geq 65(\%)$ & 13.2 & 8.0 & 14.3 & & & \\
\hline Male, gender, $\%$ & 55.3 & 51.8 & 62.7 & 0.07 & 0.37 & 0.04 \\
\hline Geographic region & & & & 0.36 & 0.78 & 0.15 \\
\hline Utah (\%) & 95.3 & 95.0 & 95.1 & & & \\
\hline Idaho (\%) & 3.4 & 2.0 & 3.7 & & & \\
\hline Other (\%) & 1.0 & 3.0 & 1.4 & & & \\
\hline Insurance line & & & & 0.06 & 0.21 & 0.04 \\
\hline Commercial (\%) & 86.4 & 94.5 & 88.3 & & & \\
\hline Medicare (\%) & 10.2 & 4.5 & 9.6 & & & \\
\hline Medicaid (\%) & 3.4 & 1.0 & 2.2 & & & \\
\hline \multicolumn{7}{|l|}{ Baseline Alc (\%) } \\
\hline Mean (SD) & $9.55(1.97)$ & $8.42(1.27)$ & $8.49(1.39)$ & $<0.01$ & $<0.01$ & 0.48 \\
\hline Alc $\geq 7.0 \%$ to $<9.0 \%$ & 46.8 & 72.9 & 72.7 & $<0.01$ & $<0.01$ & 1.00 \\
\hline Alc $\geq 9.0 \%$ & 53.2 & 27.1 & 27.3 & & & \\
\hline CCI ${ }^{\mathrm{d}}$ score, mean (SD) & $1.90(1.31)$ & $1.68(1.21)$ & $1.66(1.10)$ & $<0.01$ & 0.01 & 0.76 \\
\hline $1(\%)$ & 54.9 & 61.8 & 63.0 & 0.11 & 0.02 & 0.83 \\
\hline $2(\%)$ & 22.0 & 23.6 & 21.2 & & & \\
\hline $3(\%)$ & 10.8 & 6.5 & 7.9 & & & \\
\hline$\geq 4(\%)$ & 12.2 & 8.0 & 7.9 & & & \\
\hline DCSI $^{\mathrm{d}}$ score, mean (SD) & $0.79(1.29)$ & $0.60(1.04)$ & $0.59(1.02)$ & 0.01 & 0.02 & 0.89 \\
\hline $0(\%)$ & 62.0 & 64.3 & 65.3 & 0.08 & 0.01 & 0.98 \\
\hline $1(\%)$ & 16.6 & 21.1 & 19.8 & & & \\
\hline $2(\%)$ & 10.2 & 9.0 & 9.0 & & & \\
\hline$\geq 3(\%)$ & 11.2 & 5.5 & 5.9 & & & \\
\hline Baseline treatment & & & & $<0.01$ & $<0.01$ & 0.32 \\
\hline MET +SU (\%) & 65.4 & 54.8 & 50.1 & & & \\
\hline MET + DPP-4 (\%) & 22.7 & 26.1 & 31.1 & & & \\
\hline MET + TZD (\%) & 4.1 & 8.5 & 9.0 & & & \\
\hline SU + DPP-4 (\%) & 5.1 & 6.5 & 4.1 & & & \\
\hline Other (\%) & 2.7 & 4.0 & 5.6 & & & \\
\hline \multicolumn{7}{|c|}{ 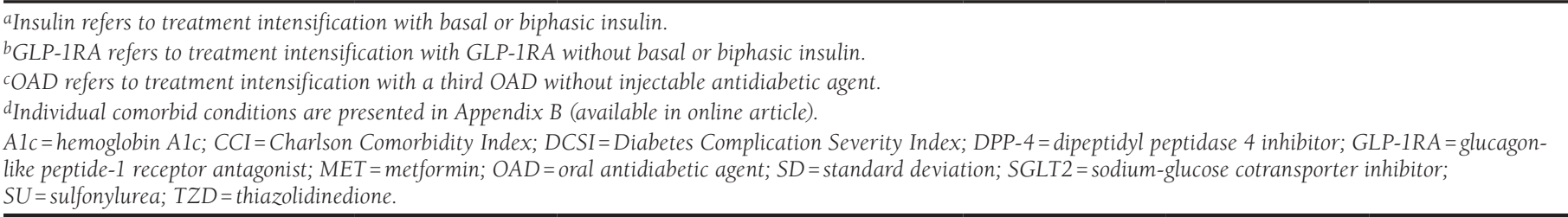 } \\
\hline
\end{tabular}

glycemic control on treatment outcomes. Once adjusted for the baseline Alc measure, the model predictability based on Akaike Information Criteria (AIC) values and likelihood ratio test results did not improve with the inclusion of any additional covariates. To alleviate concerns of residual confounding, the odds ratio (OR) was further adjusted for age, gender, baseline treatment group, and CCI score in a multivariate regression model. Covariate selection between the CCI and DCSI scores was based on the AIC and Bayesian Information Criterion, where the lower score represents the better model fit.
Glycemic control could be influenced by the length of follow-up from the intensification to the outcome assessment, and length of follow-up type could differ by the class of agent prescribed to intensify therapy. To address this potential bias, the analytic cohort was further stratified by the time to followup Alc measurement from 60 to 120 days from the intensification and $>120$ days after intensification. The 120-day cut-off was determined based on the median follow-up (i.e., 119 days) from the overall cohort. Therefore, the analysis maintained a similar number of patients between the 2 subcohorts and was identically powered for the 2 subcohorts. 

Days to Follow-Up A1c from Treatment Intensification by Type of Treatment Intensification

\begin{tabular}{|c|c|c|c|c|c|c|}
\hline & \multirow[b]{2}{*}{$\begin{array}{l}\text { Insulin } \\
\mathrm{n}=295\end{array}$} & \multirow[b]{2}{*}{$\begin{array}{c}\text { GLP-1RA } \\
\mathrm{n}=199\end{array}$} & \multirow[b]{2}{*}{$\begin{array}{l}\text { OAD only } \\
\mathbf{n}=732\end{array}$} & \multicolumn{3}{|c|}{$P$ Value } \\
\hline & & & & 3 Arms & $\begin{array}{l}\text { Insulin } \\
\text { vs. Other }\end{array}$ & $\begin{array}{l}\text { GLP-1RA } \\
\text { vs. OAD }\end{array}$ \\
\hline Alc at baseline (mean, SD) & $9.56(1.97)$ & $8.42(1.27)$ & $8.49(1.39)$ & $<0.01$ & $<0.01$ & 0.48 \\
\hline Alc at follow-up (60-365 days after treatment intensification; mean, SD) & $8.54(1.89)$ & $7.80(1.71)$ & $7.96(1.54)$ & $<0.01$ & $<0.01$ & 0.20 \\
\hline Change in Alc (mean, SD) & $-1.02(2.08)$ & $-0.62(1.59)$ & $-0.52(1.60)$ & $<0.01$ & $<0.01$ & 0.45 \\
\hline Baseline Alc < $9.0 \%(\%)$ & 46.8 & 72.9 & 72.7 & $<0.01$ & $<0.01$ & 1.00 \\
\hline Follow-up Alc $<7.0 \%(\%)$ & 18.6 & 38.7 & 25.5 & $<0.01$ & $<0.01$ & $<0.01$ \\
\hline Follow-up < $9.0 \%$ (\%) & 65.4 & 78.4 & 79.9 & $<0.01$ & $<0.01$ & 0.73 \\
\hline Days from baseline to treatment intensification (mean, SD) & $111.6(112.2)$ & $121.9(114.0)$ & $102.8(108.4)$ & 0.13 & 0.52 & 0.03 \\
\hline Days from treatment intensification to follow-up (mean, SD) & $141.3(69.7)$ & $138.5(71.0)$ & $145.0(70.5)$ & 0.35 & 0.63 & 0.25 \\
\hline
\end{tabular}

Alc = hemoglobin A1c; GLP-1RA = glucagon-like peptide-1 receptor antagonist; OAD=oral antidiabetic agent; SD=standard deviation.

\section{Results}

\section{Cohort Extraction and Baseline Characteristics by Treatment Intensification}

The analytic cohort included 1,226 patients who met the baseline inclusion criteria and had a record of treatment intensification and an outcome Alc measure. Of the cohort, 54.6\% ( $n=669$ ) received MET + SU during the 1 -year baseline period, and $28.3 \%(n=347)$ received MET + DPP-4. Baseline treatment with MET + TZD and SU+DPP-4 accounted for $7.7 \%(n=95)$ and $4.7 \%(n=58)$ of the cohort, respectively (Table 1$)$.

Treatment intensification with insulin was observed in 295 (24.1\%) patients, while 199 (16.2\%) patients were prescribed a GLP-1RA, and 732 (59.7\%) were prescribed a third OAD to intensify therapy (Table 1). GLP-1RA recipients were slightly younger with a mean age of $52.2 \pm 10.3$ years versus those receiving insulin $(53.9 \pm 11.2$ years $)$ or a third OAD $(55.5 \pm 10.0$ years). The CCI and DCSI scores were significantly higher in the insulin group (CCI: $1.90 \pm 1.31, P<0.01$; DCSI: $0.79 \pm 1.29$, $P=0.01)$ versus others.

There was no statistically significant difference in the comorbidity scores between the GLP-1RA (CCI: 1.68 \pm 1.21 ; DCSI: $0.60 \pm 1.04$ ) and the third OAD (CCI: $1.66 \pm 1.10$; DCSI: $0.59 \pm 1.02)$ groups. The number of days from treatment intensification to the follow-up Alc value was similar for the insulin, GLP-1RA, and OAD groups, with follow-up periods of 141.3, 138.5, and 145.0 days, respectively (Table 2).

More than half of the patients whose therapy was intensified with insulin had baseline Alc $\geq 9.0 \%$, whereas the corresponding proportions of patients in the GLP-1RA and third OAD groups with baseline Alc $\geq 9.0 \%$ was less than 30\% (Table 1). This accounts for the predominantly higher mean baseline Alc in patients receiving insulin (9.55\%) versus the GLP-1RA (8.42\%) or third OAD (8.49\%) intensification groups $(P<0.01)$. The number of days to treatment intensification was not statistically different $(P=0.13$, ANOVA) when the analysis was performed across the insulin (111.6 days), GLP-1RA (121.9 days), and third OAD groups (102.8 days; Table 2). However, the comparison limited to the 2 noninsulin groups showed that patients who received GLP-1RA had significantly longer time to receive the intensification than third OAD group $(P=0.03$; Table 2).

\section{Outcomes After Treatment Intensification}

In general, glycemic control in the study cohort improved from baseline to follow-up with $26.0 \%$ of the cohort achieving $\mathrm{Alc}<7.0 \%$ at follow-up (60-365 days from the treatment intensification). The proportion of patients with Alc between 7.0\% and $7.9 \%$ decreased from $40.4 \%$ to $31.6 \%$ from baseline to follow-up. Similarly, the proportion of patients with Alc between $8.0 \%$ and $8.9 \%$ dropped from $26.1 \%$ to $18.5 \%$, and the proportion with Alc $\geq 9.0 \%$ changed from $33.5 \%$ to $23.8 \%$ during the same time period (Figure 2).

Patients whose treatment was intensified with insulin experienced a significantly larger Alc reduction (-1.02\%) than those receiving a GLP-1RA $(-0.62 \%)$ or third OAD $(-0.52 \% ; P<0.01)$. However, the percentage of patients achieving $\mathrm{Alc}<7.0 \%$ and $<9.0 \%$ at follow-up was significantly lower in patients with insulin intensification (18.6\% and $65.4 \%$, respectively) compared with the GLP-1RA (38.7\% and $78.4 \%$, respectively) and third OAD groups (25.5\% and $79.9 \%$ ), respectively. This finding reflects the higher average baseline Alc in the insulin intensification group.

There was no statistically significant difference in the follow-up Alc levels between GLP-1RA (7.80\%) and third OAD (7.96\%) patients or in Alc change from baseline. On the other hand, a significantly larger proportion of patients in the GLP1RA group achieved Alc $<7.0 \%$ compared with the patients in the third OAD group (38.7\% vs. 25.5\%, P<0.01; Table 2). GLP-1RA patients were also significantly more likely to achieve Alc $<7.0 \%$ at follow-up than third OAD patients, specifically when the analysis was limited to those with a baseline Alc $<9.0 \%(\mathrm{OR}=2.29,95 \% \mathrm{CI}=1.56-3.37$; Table 3). 


\section{FIGURE 2 Distribution of Baseline and Follow-Up Alc}

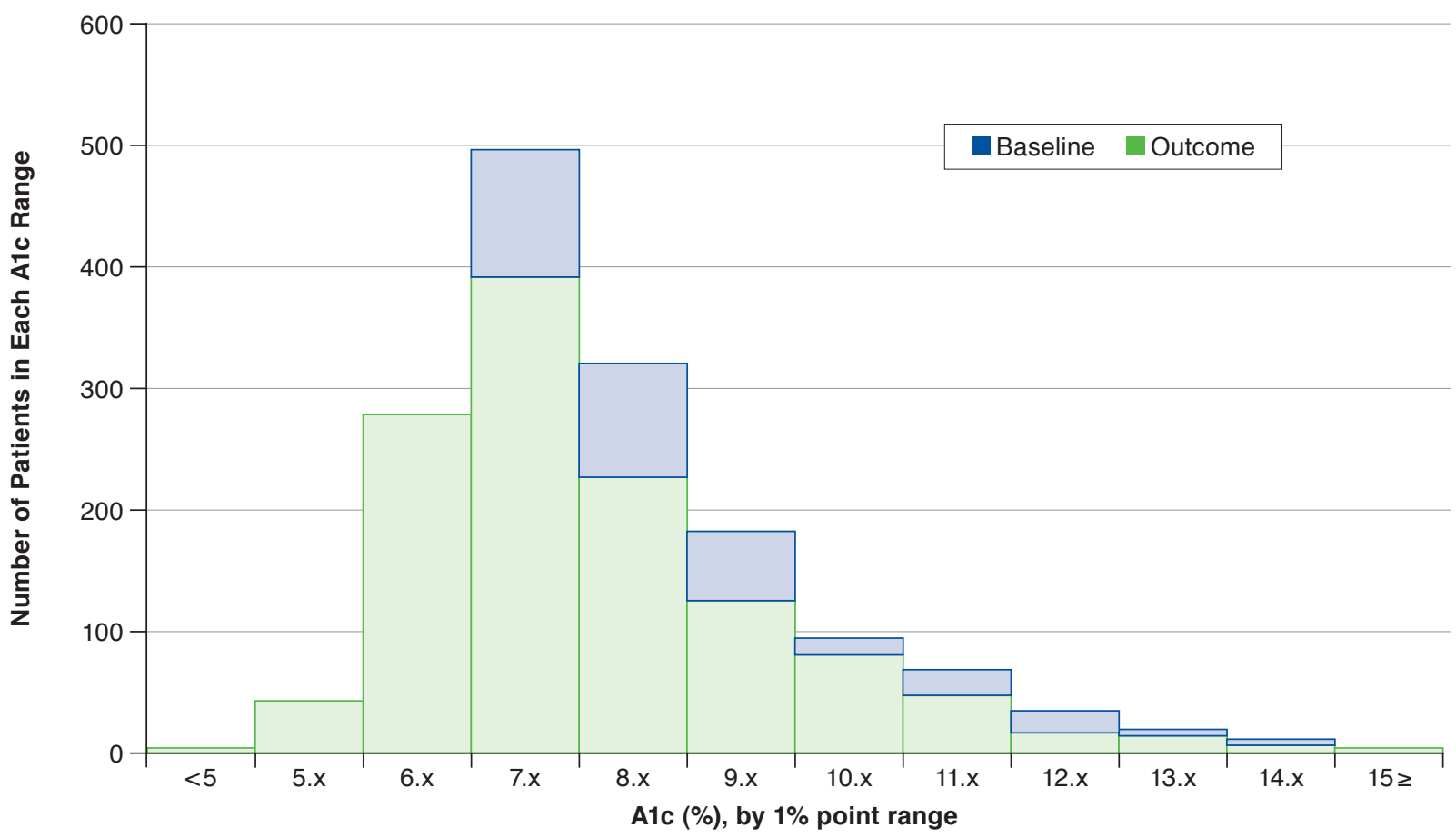

Alc =hemoglobin Alc.

The effect of insulin versus noninsulin maintained when the analysis was stratified by the time to follow-up (data not provided). In patients having the outcome Alc within 120 days, the insulin arm achieved a significantly larger Alc reduction $(-1.24 \%)$ than those receiving a GLP-1RA (-0.95\%) or third $\operatorname{OAD}(-0.69 \% ; P<0.01)$. The Alc reduction after 120 days from the insulin intensification $(-0.78 \%)$ was significantly greater $(P=0.02)$ than the reduction after the GLP-1RA $(-0.24 \%)$ or third OAD $(-0.36 \%)$.

The outcome after the intensification with GLP-1RA versus OAD was influenced by the time to follow-up. The OR of achieving Alc $<9.0 \%$ or $7.0 \%$ consistently favored GLP-1RA for the 120-day follow-up, with the point estimates between 1.87 and 2.75. In patients having the outcome after 120 days $\mathrm{OAD}$, the ORs of GLP-1RA versus OAD for the outcome Alc $<9.0 \%$ were negative and statistically significant, specifically in patients with a baseline Alc $<9.0 \% \quad(\mathrm{OR}=0.28,95 \% \mathrm{CI}=0.11-0.68$; Table 3). On the other hand, in patients with a baseline Alc $\geq 9.0 \%$, GLP-1RA was associated with better odds of the outcome Alc $<7.0 \%$ than OADs after 120 days $(\mathrm{OR}=2.22,95 \%$ $\mathrm{CI}=$ 1.24-3.98; Table 3).

\section{Discussion}

This study identified that intensification of diabetes therapy improved glycemic control outcomes in patients with suboptimally controlled glycemia (Alc $\geq 7.0 \%$ ) on 2 OADs. The proportion of patients with Alc $<7.0 \%$ increased at follow-up, while those with Alc between $7.0 \%$ and $9.0 \%$ or over $9.0 \%$ declined. Patients whose treatment was intensified with insulin had a greater mean Alc reduction than those receiving a GLP-1RA or a third OAD. However, a smaller proportion of patients in the insulin group achieved Alc $<7.0 \%$ and $<9.0 \%$ at follow-up, presumably because of higher baseline Alc levels. In general, our results are consistent with previous studies that showed a controlled Alc outcome after treatment intensification..$^{14,15,18}$ Specifically, the results are comparable in that patients who received insulin for the treatment intensification achieved a substantial drop in Alc. ${ }^{18}$

The study findings also indicate that patients and providers turn to oral agents more often than either insulin or a GLP1RA. The proportion of patients in our study who continued to use OADs (60\%) was similar to a previous study. ${ }^{18}$ The choice to use insulin was presumably determined by the baseline Alc, with a difference in baseline Alc between insulin versus noninsulin groups exceeding $1 \%$ point. Before treatment intensification, more than half of the patients in the insulin intensification group were classified as "poorly controlled" at baseline, based on the NCQA comprehensive diabetes care recommendation. ${ }^{7}$ At follow-up, this proportion was decreased 
TABLE 3 Odds of Achieving Alc $<7.0$ and $<9.0$ for GLP-1RA Versus Third OADs per Multivariable Logistic Regression Analyses

\begin{tabular}{|c|c|c|c|c|}
\hline \multirow[b]{2}{*}{ Outcome } & \multirow[b]{2}{*}{ By Baseline Alc } & \multicolumn{3}{|c|}{ Time to Outcome Alc } \\
\hline & & $\begin{array}{c}\text { 60-365 Days, } \mathrm{n}=1,226 \\
\text { OR }(95 \% \mathrm{CI})\end{array}$ & $\begin{array}{l}\text { 60-120 Days, } \mathrm{n}=620 \\
\text { OR }(95 \% \mathrm{CI})\end{array}$ & $\begin{array}{c}121-365 \text { Days, } \mathrm{n}=606 \\
\text { OR }(95 \% \mathrm{CI})\end{array}$ \\
\hline \multirow{4}{*}{ Alc $<9.0 \%$} & Alla $^{\mathrm{a}}$ & $2.05(1.45-2.90)$ & $2.46(1.11-5.47)$ & $0.48(0.28-0.83)$ \\
\hline & Allb & $2.28(1.55-3.36)$ & $2.63(0.89-7.76)$ & $0.67(0.32-1.42)$ \\
\hline & Baseline Alc $\geq 9.0 \%$ & $1.24(0.53-2.87)$ & $2.62(0.88-7.75)$ & $0.66(0.31-1.41)$ \\
\hline & Baseline Alc $<9.0 \%$ & $2.29(1.56-3.37)$ & $2.75(0.78-9.69)$ & $0.28(0.11-0.68)$ \\
\hline \multirow{4}{*}{$\mathrm{Alc}<7.0 \%$} & Alla & $2.05(1.45-2.90)$ & $2.03(1.27-3.23)$ & $1.90(1.13-3.18)$ \\
\hline & Allb & $2.28(1.55-3.36)$ & $2.10(1.26-3.50)$ & $2.20(1.23-3.94)$ \\
\hline & Baseline Alc $\geq 9.0 \%$ & $1.24(1.24-2.87)$ & $2.07(1.24-3.45)$ & $2.22(1.24-3.98)$ \\
\hline & Baseline Alc $<9.0 \%$ & $2.29(1.56-3.37)$ & $1.87(0.55-6.39)$ & $1.01(0.30-3.45)$ \\
\hline
\end{tabular}

Note: All ORs were adjusted for age (<65 vs. $\geq 65$ years), gender, baseline treatment group, and Charlson Comorbidity Index score (1, 2, 3, or $\geq 4)$.

a Model also adjusted for baseline Alc $\geq 9.0 \%$ versus $<9.0 \%$.

${ }^{b}$ Model also adjusted for baseline Alc $\geq 9.0 \%$ versus $<9.0 \%$ and an interaction term of baseline Alc and the intensification group.

Alc =hemoglobin Alc; CI= confidence interval; GLP-1RA=glucagon-like peptide-1 receptor antagonist; OAD=oral antidiabetic agent; OR=odds ratio.

to $34.6 \%$ in patients receiving insulin. The insulin group also experienced a clinically substantial reduction in Alc (over $1 \%$ point), reflecting insulin's potency when dosed appropriately. However, regression and stratified analyses suggested that confounding by baseline Alc was considerable and that we were unable to fully control for baseline diabetes severity. Thus, our results comparing intensification with insulin versus GLP-1RA or OADs in our target population may be biased. Nonetheless, the significant and substantial Alc reduction from baseline to follow-up with insulin therapy sufficiently demonstrates the relative benefit of insulin intensification in patients whose glycemia is poorly or not optimally controlled with a regimen of 2 OADs.

GLP-1RA, a relatively newer strategy compared with insulin or OADs in general, accounted for $16 \%$ of the treatment intensifications. Patients whose treatment was intensified with a GLPIRA tended to be younger and female with commercial coverage than patients whose treatment was intensified with a third OAD. When it comes to the other clinical characteristics, however, there was no significant difference in the baseline Alc or comorbidity profiles between the GLP-1RA and third OAD cohorts.

Among the noninsulin patients, Alc reduction with intensification did not differ between GLP-1RA and a third OAD nor the odds of achieving Alc $<9.0 \%$. However, the odds of achieving Alc $<7.0 \%$ favored GLP-1RA relative to a third OAD, particularly in patients with a baseline Alc between $7.0 \%$ and $9.0 \%$. The association between intensification with GLP-1RA versus an OAD was further influenced by the time to follow-up Alc. For example, GLP-1RA was associated with higher odds of having outcome Alc $<7.0$, as well as Alc $<9.0$ within 120 days from the intensification across the different levels of Alc at baseline. The lower Alc with the use of GLP-1RA did not replicate in patients with baseline Alc $\geq 9.0 \%$ and whose follow-up Alc occurred more than 120 days after intensification.
Multiple prospective studies have identified more favorable glycemic control outcomes with GLP-1RA versus OADs, including MET, SU, and TZD. However, only a limited number of studies demonstrated the superiority of GLP-1RA over OADs in a real-world setting. ${ }^{21}$ Our study generally supports the use of GLP-1RA as third-line therapy after failure on 2 OADs based on overall treatment goals and patient characteristics. However, our mixed findings from the subgroup analysis demonstrated that the benefits of GLP-1RA may be greatest in patients with higher baseline Alc and whose glycemic control is monitored per treatment guidelines, which suggest that Alc should be measured every 90 days in patients with inadequate glycemic control and after treatment changes. ${ }^{22}$

An important finding of this study is that even with intensification, there was an average delay of 3.5 months between baseline Alc $>7.0 \%$ and treatment intensification. Practice recommendations encourage timely intensification when treatment fails to reduce the risk of poor outcomes, thus, managed care organizations have an opportunity to help improve patient care and outcomes by encouraging timely intensification. ${ }^{16}$ Successful interventions will need to address barriers to treatment intensification. While this study was not designed to assess these barriers, this topic is worthy of further investigation. ${ }^{15}$

In recognizing the value of optimizing diabetes care, over $90 \%$ of U.S. health plans have adopted the NCQA-HEDIS measures, including comprehensive diabetes care measures. ${ }^{23}$ In addition to recommended processes of care for diabetes (e.g., annual eye and foot exams and Alc testing), the comprehensive diabetes care set includes Alc outcomes as a measure of diabetes care performance for health plans overall, as well as for providers. As a further incentive to improve diabetes outcomes and reduce costs, public and private payers are basing a component of provider reimbursement on diabetes quality measures performance, such as foot and eye screenings on a 
regular basis. ${ }^{24}$ Further, the Centers for Medicare \& Medicaid Services (CMS) assesses Alc $<9.0 \%$ as a performance measure in the Quality Payment Program for accountable care organizations and group practices, as well as in the Medicare Star Quality Rating System. ${ }^{24-26}$ Thus, high performance on diabetes outcomes-based quality measures is an important goal for health plans and is considered when prioritizing patient interventions, such as targeting patients with poorly controlled diabetes (Alc $\geq 9.0 \%$ ) for education and treatment intensification.

While there is solid trial-based evidence supporting the use of diabetes agents and informing place in therapy for a given patient, outcomes are not regularly reported in a way that can shed light on whether interventions and treatment strategies will have a positive influence on performance to diabetes care measures. This study has elucidated the outcomes of treatment intensification in patients whose Alc levels failed to attain optimal control after therapy with 2 OADs from a quality measures perspective. Thus, the information from this study will give health plans and providers information on the value of treatment intensification in terms of improving patient outcomes and improving diabetes care performance in the eyes of employers and CMS as the ultimate health care purchasers and payer.

\section{Limitations}

Any interpretation of this study is accompanied by study limitations. First, we used health plan claims data that are augmented by Alc laboratory records. We did not have access to additional clinical data or information on patient behaviors that could influence treatment selection and patient outcomes (e.g., weight/body mass index, blood pressure, and adherence to diet and exercise recommendations). This limitation is partially addressed by a robust linkage of claims and medical records data.

Second, we were unable to completely control for differences in baseline Alc between insulin and the other treatment intensification groups. Future retrospective studies may consider matching techniques to more effectively isolate the effect of treatment on outcomes, but such techniques may introduce selection bias and reduce generalizability.

Third, treatment intensification was determined by a claim for a new class of diabetes medication, but we did not determine if the newly prescribed class represented an add-on or a switch in therapy. We also did not identify dose escalations as an alternate form of treatment intensification. In addition, we did not assess medication compliance, which could affect treatment selection and outcomes and possibly be a determinant of time to intensification.

Fourth, we limited follow-up assessment to the first Alc measure 60-365 days after treatment intensification versus using all available follow-up Alc data. Our future research will consider the longitudinal changes in Alc after the treatment intensification to assess time to goal and sustainability of goal attainment by treatment intensification strategy.

Finally, this study was subject to general limitations in the use of claims data, including the potential for miscoding, unmeasured confounders, and situations when medications are not reimbursed through the health plan, such as medication samples and cash purchases of low-cost generics.

\section{Conclusions}

This study provided insight into current treatment intensification patterns for patients with T2DM whose regimen is intensified after failing to attain glycemic control while on 2 OADs. We identified that treatment intensification is associated with improved glycemic control, as expected. Further, improved glycemic control translates to better performance relative to diabetes quality measures in this study population. Health plans should encourage appropriate treatment intensification in patients with poorly controlled diabetes while on multiple oral agents. Future research to provide data on glycemic control sustainability and barriers to treatment intensification will help to optimize health plan efforts to reduce therapeutic inertia.

\section{Authors}

KIBUM KIM, BPharm, MSc, PhD; SUDHIR UNNI, PhD, MBA; CARRIE MCADAM-MARX, RPh, MS, PhD; and DIANA BRIXNER, RPh, PhD, FAMCP, Department of Pharmacotherapy, University of Utah, Salt Lake City. SHEILA M. THOMAS, PharmD; KIMBERLY L. STERLING, PharmD, MS; and BRYAN JOHNSTONE, PhD, Sanofi, Bridgewater, New Jersey. CODY J. OLSEN, PharmD, BCPS, and MATT MITCHELL, PharmD, MBA, FAMCP, SelectHealth, Murray, Utah.

AUTHOR CORRESPONDENCE: Diana Brixner, RPh, PhD, FAMCP, 30 South 2000 East, Salt Lake City, UT 84112. Tel.: 801.587.9639; E-mail: diana.brixner@utah.edu.

\section{DISCLOSURES}

Research funding was provided by Sanofi to the Pharmacotherapy Outcomes Research Center at the University of Utah and SelectHealth to conduct this study. Thomas, Sterling, and Johnstone are employees and stock/shareholders of Sanofi. Kim, Unni, McAdam-Marx, and Brixner are employees of the Department of Pharmacotherapy at the University of Utah. Brixner also has served as an advisory board member and presenter for Sanofi. McAdam-Marx also reports grants to the Department of Pharmacotherapy, University of Utah, from AstraZeneca and Janssen, outside of the submitted work. Olsen is employed by SelectHealth.

Part of the results of this study was presented at the Academy of Managed Care \& Specialty Pharmacy Annual Meeting 2018 in Boston, MA, during April 23-26, 2018 


\section{REFERENCES}

1. American Diabetes Association. Economic costs of diabetes in the U.S. in 2012. Diabetes Care. 2013;36(4):1033-46.

2. Centers for Disease Control and Prevention. National diabetes statistics report, 2017: estimates of diabetes and its burden in the United States. 2017. Available at: https://www.cdc.gov/diabetes/pdfs/data/statistics/nationaldiabetes-statistics-report.pdf. Accessed January 8, 2019.

3. Fowler MJ. Microvascular and macrovascular complications of diabetes. Clin Diabetes. 2008;26(2):77-82.

4. Monami M, Adalsteinsson JE, Desideri CM, Ragghianti B, Dicembrini I, Mannucci E. Fasting and post-prandial glucose and diabetic complication: a meta-analysis. Nutr Metab Cardiovasc Dis. 2013;23(7):591-98.

5. Stratton IM, Adler AI, Neil HA, et al. Association of glycaemia with macrovascular and microvascular complications of type 2 diabetes (UKPDS 35): prospective observational study. BMJ. 2000;321(7258):405-12.

6. Shi L, Ye X, Lu M, et al. Clinical and economic benefits associated with the achievement of both $\mathrm{HbA}(\mathrm{lc})$ and LDL cholesterol goals in veterans with type 2 diabetes. Diabetes Care. 2013;36(10):3297-304.

7. National Committee for Quality Assurance. Comprehensive diabetes care. October 31, 2017. Available at: http://www.ncqa.org/report-cards/healthplans/state-of-health-care-quality/2017-table-of-contents/diabetes-care. Accessed January 8, 2019.

8. Dickerson LM, Ables AZ, Everett CJ, et al. Measuring diabetes care in the national interdisciplinary primary care practice-based research network (NIPC-PBRN). Pharmacotherapy. 2011;31(1):23-30.

9. Planas LG, Crosby KM, Farmer KC, Harrison DL. Evaluation of a diabetes management program using selected HEDIS measures. J Am Pharm Assoc (2003). 2012;52(6):e130-38

10. Ragucci KR, Fermo JD, Wessell AM, Chumney EC. Effectiveness of pharmacist-administered diabetes mellitus education and management services. Pharmacotherapy. 2005;25(12):1809-16.

11. Cavero-Redondo I, Peleteiro B, Álvarez-Bueno C, Rodriguez-Artalejo F, Martínez-Vizcaíno V. Glycated haemoglobin Alc as a risk factor of cardiovascular outcomes and all-cause mortality in diabetic and non-diabetic populations: a systematic review and meta-analysis. BMJ Open. 2017;7(7):e015949.

12. Ali MK, Bullard KM, Saaddine JB, Cowie CC, Imperatore G, Gregg EW. Achievement of goals in U.S. diabetes care, 1999-2010. N Engl J Med. 2013;368(17):1613-24.

13. Pantalone KM, Wells BJ, Chagin KM, et al. Intensification of diabetes therapy and time until AlC goal attainment among patients with newly diagnosed type 2 diabetes who fail metformin monotherapy within a large integrated health system. Diabetes Care. 2016;39(9):1527-34.
14. Fu AZ, Sheehan JJ. Treatment intensification for patients with type 2 diabetes and poor glycaemic control. Diabetes Obes Metab. 2016;18(9):892-98. 15. Fu AZ, Sheehan JJ. Change in HbAlc associated with treatment intensification among patients with type 2 diabetes and poor glycemic control. Curr Med Res Opin. 2017;33(5):853-58.

16. Garber AJ, Abrahamson MJ, Barzilay JI, et al. Consensus statement by the American Association of Clinical Endocrinologists and American College of Endocrinology on the comprehensive type 2 diabetes management algorithm - 2018 executive summary. Endocr Pract. 2018;24(1):91-120.

17. American Diabetes Association. Pharmacologic approaches to glycemic treatment: standards of medical care in diabetes-2018. Diabetes Care. 2018;41(Suppl 1):S73-S85.

18. Levin PA, Wei W, Zhou S, Xie L, Baser O. Outcomes and treatment patterns of adding a third agent to 2 OADs in patients with type 2 diabetes. J Manag Care Pharm. 2014;20(5):501-12. Available at: https://www.jmcp.org/ doi/10.18553/jmcp.2014.20.5.501.

19. Glasheen WP, Renda A, Dong Y. Diabetes Complications Severity Index (DCSI)-update and ICD-10 translation. J Diabetes Complications. 2017;31(6):1007-13

20. Quan H, Sundararajan V, Halfon P, et al. Coding algorithms for defining comorbidities in ICD-9-CM and ICD-10 administrative data. Med Care. 2005;43(11):1130-39.

21. Levin PA, Nguyen H, Wittbrodt ET, Kim SC. Glucagon-like peptide-1 receptor agonists: a systematic review of comparative effectiveness research. Diabetes Metab Syndr Obes. 2017;10:123-39.

22. American Diabetes Association. Glycemic targets: standards of medical care in diabetes-2018. Diabetes Care. 2018;41(Suppl 1):S55-64.

23. National Committee for Quality Assurance. HEDIS and performance measurement. February 20, 2018. Available at: http://www.ncqa.org/hedisquality-measurement. Accessed January 8, 2019.

24. American Diabetes Association. Standard of medical care in diabetes2016. Diabetes Care. 2016;39(Suppl 1):S4-S5.

25. Centers for Medicare \& Medicaid Services. Medicare Shared Savings Program quality measure benchmarks for the 2015 reporting year. 2015. Available at: https://www.cms.gov/Medicare/Medicare-Fee-for-ServicePayment/sharedsavingsprogram/Downloads/MSSP-QM-Benchmarks-2015. pdf. Accessed January 8, 2019

26. Center for Medicare \& Medicaid Services. Medicare 2018 Part C $\&$ D Star Ratings Technical Notes. Draft. September 6, 2017. Available at: https://www.cms.gov/Medicare/Prescription-Drug-Coverage/ PrescriptionDrugCovGenIn/Downloads/2018-Star-Ratings-TechnicalNotes-2017_09_06.pdf. Accessed January 24, 2019. 
Influence of Treatment Intensification on A1c in Patients with Suboptimally Controlled Type 2 Diabetes After 2 Oral Antidiabetic Agents

\section{APPENDIX A Cohort Extraction Flowchart}

Insurance claims between January 1, 2010, and March 31, 2017

Commercial: 1,476K; Medicaid: 224K; Medicare: 52K

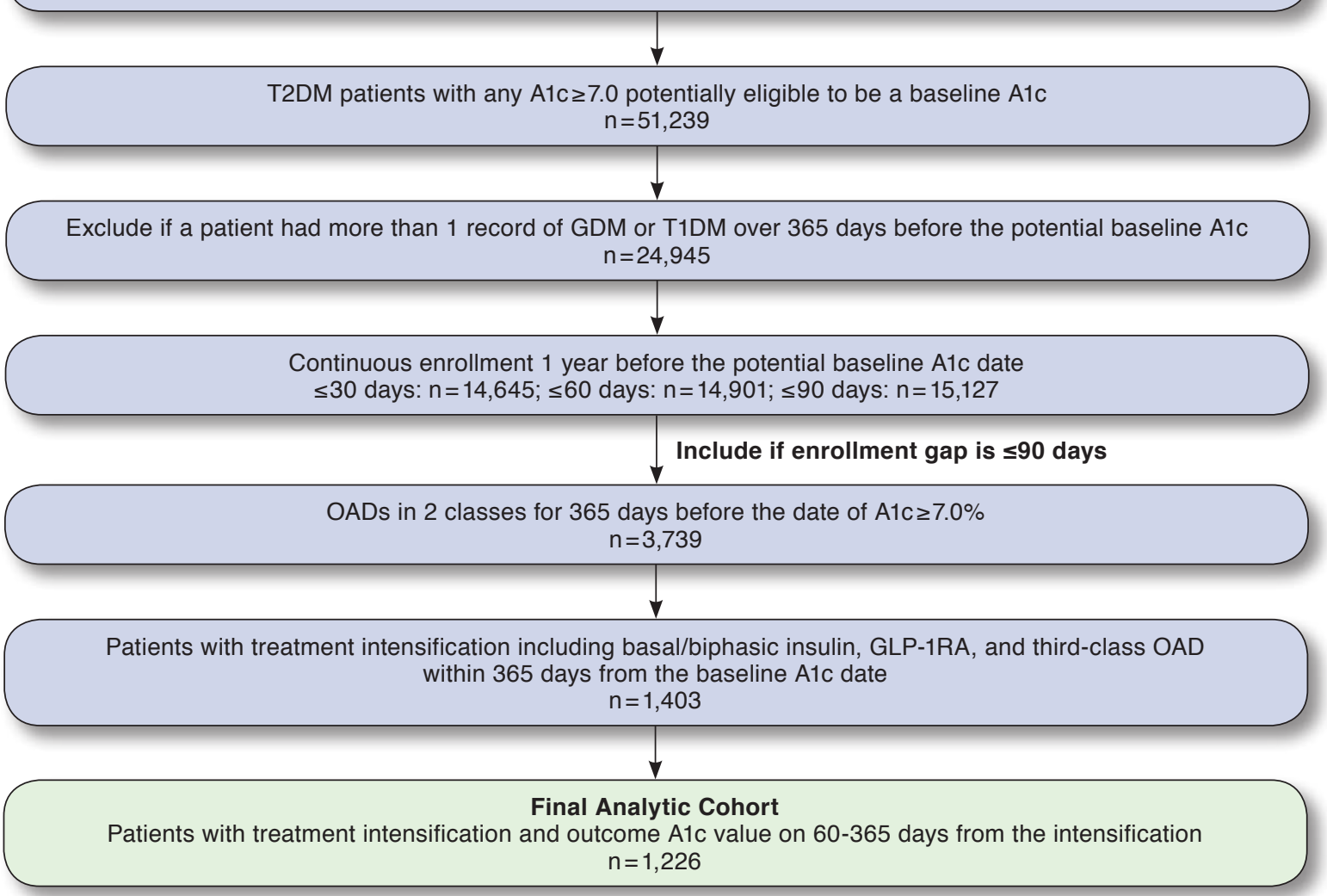

Alc =hemoglobin A1c; GDM = gestational diabetes mellitus; GLP-1RA=glucagon-like peptide 1 receptor antagonist; OAD=oral antidiabetic agent; T1DM=type 1 diabetes mellitus; T2DM= type 2 diabetes mellitus. 
Influence of Treatment Intensification on A1c in Patients with Suboptimally Controlled Type 2 Diabetes After 2 Oral Antidiabetic Agents

\section{APPENDIX B Comorbidities Based on Diagnoses Coding Used in Calculating Charlson Comorbidity Index} and Diabetes Complication Severity Index Scores

\begin{tabular}{|c|c|c|c|c|c|c|}
\hline & \multirow[b]{2}{*}{$\begin{array}{c}\text { Insulin }^{\mathrm{a}} \\
\mathrm{n}=295, \%\end{array}$} & \multirow[b]{2}{*}{$\begin{array}{l}\text { GLP-1RA }{ }^{b} \\
\mathrm{n}=199, \%\end{array}$} & \multirow[b]{2}{*}{$\begin{array}{c}\mathrm{OAD}^{\mathrm{c}} \\
\mathbf{n}=732, \%\end{array}$} & \multicolumn{3}{|c|}{$P$ Value } \\
\hline & & & & 3 Arms & $\begin{array}{l}\text { Insulin } \\
\text { vs. Other }\end{array}$ & $\begin{array}{l}\text { GLP-1RA } \\
\text { vs. OAD }\end{array}$ \\
\hline \multicolumn{7}{|l|}{ Comorbidities in $\mathrm{CCI}^{20}$} \\
\hline History of myocardial infarction & 1.7 & 2.0 & 1.8 & 0.95 & 1.00 & 0.77 \\
\hline Congestive heart failure & 5.1 & 2.5 & 3.8 & 0.34 & 0.30 & 0.50 \\
\hline Peripheral vascular disorder & 2.0 & 1.5 & 2.0 & 0.92 & 1.00 & 0.78 \\
\hline Cerebrovascular disorder & 2.4 & 1.5 & 1.4 & 0.51 & 0.29 & 0.75 \\
\hline Dementia & 0.0 & 0.0 & 0.5 & 0.49 & 0.58 & 0.58 \\
\hline Pulmonary disorder & 12.9 & 13.6 & 11.1 & 0.52 & 0.61 & 0.39 \\
\hline Rheumatic & 1.4 & 1.0 & 1.1 & 0.93 & 0.75 & 1.00 \\
\hline Peptic ulcer & 1.0 & 0.0 & 0.8 & 0.45 & 0.45 & 0.35 \\
\hline Hepatic disorder, mild & 6.8 & 7.0 & 7.4 & 0.95 & 0.88 & 1.00 \\
\hline Diabetes with complication & 17.6 & 16.1 & 14.3 & 0.39 & 0.25 & 0.61 \\
\hline Para/hemiplegia & 0.3 & 0.0 & 0.1 & 0.64 & 0.42 & 1.00 \\
\hline Renal disorder & 9.5 & 5.0 & 5.7 & 0.05 & 0.02 & 0.83 \\
\hline Cancer/malignancy & 8.5 & 5.0 & 3.8 & 0.01 & 0.00 & 0.58 \\
\hline Hepatic disorder, moderate to severe & 0.3 & 0.5 & 0.1 & 0.35 & 0.56 & 0.38 \\
\hline Metastatic cancer & 0.3 & 0.5 & 0.4 & 1.00 & 1.00 & 1.00 \\
\hline HIV/AIDS & 0.0 & 0.0 & 0.0 & N/A & N/A & N/A \\
\hline \multicolumn{7}{|l|}{ Comorbidities in DCSI ${ }^{\mathrm{d}, 19}$} \\
\hline Ophthalmic complication (any) & 6.1 & 5.0 & 4.8 & 0.67 & 0.47 & 1.00 \\
\hline Ophthalmic complication (severe) & 1.0 & 0.5 & 1.2 & 0.73 & 0.57 & 0.57 \\
\hline Nephropathy (any) & 14.2 & 8.5 & 9.8 & 0.06 & 0.03 & 0.68 \\
\hline Nephropathy (severe) & 7.5 & 5.5 & 4.5 & 0.12 & 0.07 & 0.35 \\
\hline Neuropathy & 19.7 & 18.6 & 16.0 & 0.31 & 0.24 & 0.44 \\
\hline Cerebrovascular disease (any) & 1.7 & 1.5 & 1.2 & 0.74 & 0.57 & 0.73 \\
\hline Cerebrovascular disease (severe) & 1.4 & 1.5 & 1.1 & 0.78 & 0.46 & 0.77 \\
\hline Cardiovascular disease (any) & 10.2 & 8.5 & 11.5 & 0.47 & 0.84 & 0.30 \\
\hline Cardiovascular disease (severe) & 4.7 & 3.5 & 4.6 & 0.77 & 0.81 & 0.50 \\
\hline Peripheral vascular disease (any) & 5.1 & 3.0 & 2.3 & 0.07 & 0.04 & 0.61 \\
\hline Peripheral vascular disease (severe) & 4.4 & 3.0 & 1.4 & 0.03 & 0.03 & 0.13 \\
\hline Metabolic disease (any) & 1.4 & 0.5 & 0.5 & 0.40 & 0.23 & 1.00 \\
\hline Metabolic disease (severe) & 1.0 & 0.5 & 0.3 & 0.45 & 0.22 & 0.70 \\
\hline
\end{tabular}

ansulin refers to treatment intensification with basal or biphasic insulin.

${ }^{b}$ GLP-1RA refers to treatment intensification with GLP-1RA without basal or biphasic insulin.

${ }^{C} O A D$ refers to treatment intensification with a third $O A D$ without injectable antidiabetic agent.

${ }^{d}$ Complication (any), the number of patients having a record of the condition over the baseline that adds any point to the DSCI calculation; complication (severe), the number of patients having a record of the condition that needs to add 2 points to the DSCI calculation.

$C C I=$ Charlson Comorbidity Index; DCSI = Diabetes Complication Severity Index; GLP-1RA=glucagon-like peptide-1 receptor antagonist; N/A =not applicable; OAD = oral antidiabetic agent. 